\title{
ASYMPTOTIC GROWTH OF SATURATED POWERS AND EPSILON MULTIPLICITY
}

\author{
Steven Dale Cutkosky
}

\section{Introduction}

In this paper, we study the growth of saturated powers of modules. In the case of an ideal $I$ in a local $\operatorname{ring}(R, \mathfrak{m})$, the saturation of $I^{k}$ in $R$ is

$$
\left(I^{k}\right)^{\text {sat }}=I^{k}:_{R} \mathfrak{m}^{\infty}=\cup_{n=1}^{\infty} I^{k}:_{R} \mathfrak{m}^{n} .
$$

There are examples showing that the algebra of saturated powers of $I, \bigoplus_{k>0}\left(I^{k}\right)^{\text {sat }}$ is not a finitely generated $R$-algebra; for instance, in many cases the saturated powers are the symbolic powers. As such, it cannot be expected that the "Hilbert function", giving the length of the $R$-module $\left(I^{k}\right)^{\text {sat }} / I^{k}$, is very well behaved for large $k$. However, it can be shown that it is bounded above by a polynomial in $k$ of degree $d$, where $d$ is the dimension of $R$. We show that in many cases, there is a reasonable asymptotic behavior of this length.

Suppose that $(R, \mathfrak{m})$ is a Noetherian local domain of dimension $d \geq 1$. Let $L$ be the quotient field of $R$. Let $\lambda(M)$ denote the length of an $R$-module $M$. Let $F$ be a finitely generated free $R$-module, and let $E$ be a submodule of $F$ of rank $e$. Let $S=R[F]=\operatorname{Sym}(F)=\bigoplus_{k \geq 0} F^{k}$ and let $R[E]=\bigoplus_{k \geq 0} E^{k}$ be the $R$-subalgebra of $S$ generated by $E$. Let

$$
E^{k}:_{F^{k}} \mathfrak{m}^{\infty}=\cup_{n=1}^{\infty} E^{k}:_{F^{k}} \mathfrak{m}^{n}
$$

denote the saturation of $E^{k}$ in $F^{k}$. We prove the following theorem:

Theorem 1.1. Suppose that $(R, \mathfrak{m})$ is a local domain of depth $\geq 2$ which is essentially of finite type over a field $K$ of characteristic zero (or over a perfect field $K$ such that $R / \mathfrak{m}$ is algebraic over $K)$. Let $d$ be the dimension of $R$. Suppose that $E$ is a rank $e$ submodule of a finitely generated free $R$-module $F$. Then the limit

$$
\lim _{k \rightarrow \infty} \frac{\lambda\left(E^{k}:_{F^{k}} \mathfrak{m}^{\infty} / E^{k}\right)}{k^{d+e-1}} \in \mathbb{R}
$$

exists.

The conclusions of this theorem follow from Theorem 3.2 and Remark 3.3.

Theorem 1.1 is proven in the case when $E=I$ is a homogeneous ideal and $R$ is a standard graded normal $K$-algebra in our paper [3] with Hà, Srinivasan and Theodorescu. The theorem is proven with the additional assumptions that $R$ is regular, $E=I$ is an ideal in $F=R$, and the singular locus of $\operatorname{Spec}(R / I)$ is $\mathfrak{m}$ in our paper [4] with Herzog and Srinivasan. Kleiman [13] has proven Theorem 1.1 in the case that $E$ is

Received by the editors September 29, 2010.

Partially supported by NSF. 
a direct summand of $F$ locally at every nonmaximal prime of $R$. The theorem is proven for $E$ of low analytic deviation in [4], for the case of ideals, and by Ulrich and Validashti [19] for the case of modules; in the case of low analytic deviation, the limit is always zero. A generalization of this problem to the case of saturations with respect to non $\mathfrak{m}$-primary ideals is investigated by Herzog, Puthenpurakal and Verma in [10]; they show that an appropriate limit exists for monomial ideals.

An example in [3] shows that even in the case when $E$ is an ideal $I$ in a regular local ring $R$, the limit may be irrational.

An important technique in the proof of Theorem 1.1 is to use a theorem of Lazarsfeld [14] showing that the volume of a line bundle on a complex projective variety can be expressed as a limit of numbers of global sections of powers of the line bundle; Lazarsfeld's theorem is deduced from an approximation theorem of Fujita [6] (generalizations of Fujita's result to positive characteristic are given in [17] and [15]).

We can interpret our results in terms of local cohomology. Let $F_{L}^{k}=F^{k} \otimes_{R} L$, where $L$ is the quotient field of $R$, so that we have natural embeddings $E^{k} \subset F^{k} \subset F_{L}^{k}$ for all $k$. We have identities

$$
H_{\mathfrak{m}}^{0}\left(F^{k} / E^{k}\right) \cong E^{k}:_{F^{k}} \mathfrak{m}^{\infty} / E^{k} \text { and } H_{\mathfrak{m}}^{1}\left(E^{k}\right) \cong E^{k}:_{F_{L}^{k}} \mathfrak{m}^{\infty} / E^{k}
$$

Further, these two modules are equal if $R$ has depth $\geq 2$.

We thus obtain the following corollary to Theorem 1.1, which shows that the epsilon multiplicity $\varepsilon(E)$ of a module, defined as a limsup in [19], actually exists as a limit.

Corollary 1.2. Suppose that $(R, \mathfrak{m})$ is a local domain of depth $\geq 2$ which is essentially of finite type over a field $K$ of characteristic zero (or over a perfect field $K$ such that $R / \mathfrak{m}$ is algebraic over $K)$. Let $d$ be the dimension of $R$. Suppose that $E$ is a rank $e$ submodule of a finitely generated free $R$-module $F$. Then the limit

$$
\lim _{k \rightarrow \infty} \frac{(d+e-1) !}{k^{d+e-1}} \lambda\left(H_{\mathfrak{m}}^{0}\left(F^{k} / E^{k}\right)\right) \in \mathbb{R}
$$

exists. Thus the epsilon multiplicity $\varepsilon(E)$ of $E$ exists as a limit.

By the above identities of local cohomology, we see that (1) is equivalent to the statement that

$$
\lim _{k \rightarrow \infty} \frac{H_{\mathfrak{m}}^{0}\left(F^{k} / E^{k}\right)}{k^{d+e-1}}=\lim _{k \rightarrow \infty} \frac{H_{\mathfrak{m}}^{1}\left(E^{k}\right)}{k^{d+e-1}} \in \mathbb{R}
$$

exists when $\operatorname{depth}(R) \geq 2$.

In Section 4 , we extend our results to domains of dimension $d \geq 2$. We prove the following extension of Theorem 1.1, which shows that the second limit of (2),

$$
\lim _{k \rightarrow \infty} \frac{H_{\mathfrak{m}}^{1}\left(E^{k}\right)}{k^{d+e-1}} \in \mathbb{R}
$$

exists when $R$ is a domain of dimension $d \geq 2$.

Theorem 1.3. Suppose that $(R, \mathfrak{m})$ is a local domain of dimension $d \geq 2$ which is essentially of finite type over a field $K$ of characteristic zero (or over a perfect field $K$ such that $R / \mathfrak{m}$ is algebraic over $K)$. Suppose that $E$ is a rank e submodule of a finitely generated free $R$-module $F$. Then the limit

$$
\lim _{k \rightarrow \infty} \frac{\lambda\left(E^{k}:_{F_{L}^{k}} \mathfrak{m}^{\infty} / E^{k}\right)}{k^{d+e-1}} \in \mathbb{R}
$$


exists.

Theorem 1.3 follows from Theorem 4.1 and equations (24) and (6). We prove that the first limit of (2),

$$
\lim _{k \rightarrow \infty} \frac{H_{\mathfrak{m}}^{0}\left(F^{k} / E^{k}\right)}{k^{d+e-1}} \in \mathbb{R}
$$

exists when $R$ is a domain of dimension $d \geq 2$ and $E$ is embedded in $F$ of rank $<d+e$. I thank Craig Huneke, Bernd Ulrich and Javid Validashti for pointing out this interesting consequence of Theorem 1.3.

Corollary 1.4. Suppose that $(R, \mathfrak{m})$ is a local domain of dimension $d \geq 2$ which is essentially of finite type over a field $K$ of characteristic zero (or over a perfect field $K$ such that $R / \mathfrak{m}$ is algebraic over $K)$. Suppose that $E$ is a rank e submodule of a finitely generated free $R$-module $F$. Suppose that $\gamma=\operatorname{rank}(F)<d+e$. Then the limits

$$
\lim _{k \rightarrow \infty} \frac{\lambda\left(E^{k}:_{F^{k}} \mathfrak{m}^{\infty} / E^{k}\right)}{k^{d+e-1}} \in \mathbb{R}
$$

and

$$
\lim _{k \rightarrow \infty} \frac{(d+e-1) !}{k^{d+e-1}} \lambda\left(H_{\mathfrak{m}}^{0}\left(F^{k} / E^{k}\right)\right) \in \mathbb{R}
$$

exist. In particular, the epsilon multiplicity $\varepsilon(E)$ of $E$ exists as a limit.

In the case when $e=1$ and $F=R$, we get the following statement.

Corollary 1.5. Suppose that $(R, \mathfrak{m})$ is a local domain of dimension $d \geq 1$ which is essentially of finite type over a field $K$ of characteristic zero (or over a perfect field $K$ such that $R / \mathfrak{m}$ is algebraic over $K$ ). Suppose that $I$ is an ideal in $R$. Let $\left(I^{k}\right)^{\text {sat }}=I^{k}:_{R} \mathfrak{m}^{\infty}$ be the saturation of $I^{k}$. Then the limit

$$
\lim _{k \rightarrow \infty} \frac{\lambda\left(\left(I^{k}\right)^{\mathrm{sat}} / I^{k}\right)}{k^{d}} \in \mathbb{R}
$$

exists.

Asymptotic polynomial like behavior of the length of extension functions is studied by Katz and Theodorescu [12], Theodorescu [18] and Crabbe, Katz, Striuli and Theodorescu [2]. By the local duality theorem, we obtain the following corollary to Theorem 1.1.

Corollary 1.6. Suppose that $(R, \mathfrak{m})$ is a Gorenstein local domain of dimension $d \geq 2$ which is essentially of finite type over a field $K$ of characteristic zero (or over a perfect field $K$ such that $R / \mathfrak{m}$ is algebraic over $K$ ). Suppose that $E$ is a rank e submodule of a finitely generated free $R$-module $F$. Then the limit

$$
\lim _{k \rightarrow \infty} \frac{\lambda\left(\operatorname{Ext}_{R}^{d}\left(F^{k} / E^{k}, R\right)\right)}{k^{d+e-1}} \in \mathbb{R}
$$

exists. 


\section{Preliminaries}

Suppose that $(R, \mathfrak{m})$ is a Noetherian local domain of dimension $d \geq 1$ with quotient field $L$. Let $\lambda_{R}(M)$ denote the length of an $R$-module $M$. When there is no danger of confusion, we will denote $\lambda_{R}(M)$ by $\lambda(M)$.

Let $F$ be a finitely generated free $R$-module of rank $\gamma$, and let $E$ be a submodule of $F$ of rank $e$. Let $S=R[F]=\operatorname{Sym}(F)=\bigoplus_{k \geq 0} F^{k}$, and let $R[E]=\bigoplus_{k \geq 0} E^{k}$ be the $R$-subalgebra of $S$ generated by $E$. Let

$$
E^{k}:_{F^{k}} \mathfrak{m}^{\infty}=\cup_{n=1}^{\infty} E^{k}:_{F^{k}} \mathfrak{m}^{n}
$$

denote the saturation of $E^{k}$ in $F^{k}$.

Let $F_{L}^{k}=F^{k} \otimes_{R} L$ (where $L$ is the quotient field of $R$ ), so that we have natural embeddings $E^{k} \subset F^{k} \subset F_{L}^{k}$ for all $k$. Let $X=\operatorname{Spec}(R), \widetilde{E^{k}}$ be the sheafification of $E$ on $X$ and let $u_{1}, \ldots, u_{s}$ be generators of the ideal $\mathfrak{m}$.

There are identities

$$
H^{0}\left(X \backslash\{\mathfrak{m}\}, \widetilde{E^{k}}\right)=\cap_{i=1}^{s}\left(E^{k}\right)_{u_{i}}=E^{k}:_{F_{L}^{k}} \mathfrak{m}^{\infty} .
$$

From the exact sequence of cohomology groups

$$
0 \rightarrow H_{\mathfrak{m}}^{0}\left(E^{k}\right) \rightarrow E^{k} \rightarrow H_{\mathfrak{m}}^{0}\left(X \backslash\{\mathfrak{m}\}, \widetilde{E^{k}}\right) \rightarrow H_{\mathfrak{m}}^{1}\left(E^{k}\right) \rightarrow 0,
$$

we deduce that we have isomorphisms of $R$-modules

$$
H_{\mathfrak{m}}^{1}\left(E^{k}\right) \cong E^{k}:_{F_{L}^{k}} \mathfrak{m}^{\infty} / E^{k}
$$

for $k \geq 0$. The same calculation for $F^{k}$ shows that

$$
H_{\mathfrak{m}}^{1}\left(F^{k}\right) \cong F^{k}:_{F_{L}^{k}} \mathfrak{m}^{\infty} / F^{k} .
$$

From the left exact local cohomology sequence

$$
0 \rightarrow H_{\mathfrak{m}}^{0}\left(F^{k} / E^{k}\right) \rightarrow H_{\mathfrak{m}}^{1}\left(E^{k}\right) \rightarrow H_{\mathfrak{m}}^{1}\left(F^{k}\right),
$$

we have that

$$
\left.H_{\mathfrak{m}}^{0}\left(F^{k} / E^{k}\right) \cong\left(E^{k}:_{F_{L}^{k}} \mathfrak{m}^{\infty}\right) \cap F^{k}\right) / E^{k}=E^{k}:_{F^{k}} \mathfrak{m}^{\infty} / E^{k} .
$$

From (6), and the fact that $F^{k}$ is a free $R$-module, we have that $H^{0}\left(X \backslash\{\mathfrak{m}\}, \widetilde{F^{k}}\right)=$ $F^{k}$ and

$$
E^{k}:_{F_{L}^{k}} \mathfrak{m}^{\infty}=E^{k}:_{F^{k}} \mathfrak{m}^{\infty} \text { if } R \text { has depth } \geq 2 .
$$

Let $E S$ be the ideal of $S$ generated by $E$. We compute the degree $n$ part of $(E S)^{n}$ from the formula

$$
\left[(E S)^{n}\right]_{n}=E^{n} .
$$

Let $R[\mathfrak{m} E]=\bigoplus_{n \geq 0}(\mathfrak{m} E)^{n}$ be the $R$-subalgebra of $S$ generated by $\mathfrak{m} E$.

Let $X=\operatorname{Spec}(R), Y=\operatorname{Proj}(R[\mathfrak{m} E])$ and $Z=\operatorname{Proj}(R[E])$.

Write $R[E]=R\left[\bar{x}_{1}, \ldots, \bar{x}_{t}\right]$ as a standard graded $R$-algebra, with $\operatorname{deg} \bar{x}_{i}=1$ for all $i$. For $1 \leq i \leq t$, let

$$
R_{i}=R\left[\frac{\bar{x}_{1}}{\bar{x}_{i}}, \ldots, \frac{\bar{x}_{t}}{\bar{x}_{i}}\right]
$$


and let $V_{i}=\operatorname{Spec}\left(R_{i}\right)$ for $1 \leq i \leq t .\left\{V_{i}\right\}$ is an affine cover of $Z$. Let $u_{1}, \ldots, u_{s}$ be generators of the ideal $\mathfrak{m}$. For $1 \leq i \leq s$ and $1 \leq j \leq t$, let

$$
R_{i, j}=R\left[\frac{u_{\alpha} \bar{x}_{\beta}}{u_{i} \bar{x}_{j}} \mid 1 \leq \alpha \leq s, 1 \leq \beta \leq t\right],
$$

and $U_{i, j}=\operatorname{Spec}\left(R_{i, j}\right)$. Then $\left\{U_{i, j}\right\}$ is an affine cover of $Y$. Since

$$
R_{j}\left[\frac{u_{1}}{u_{i}}, \ldots, \frac{u_{s}}{u_{i}}\right]=R_{i, j},
$$

we see that $Y$ is the blow up of the ideal sheaf $\mathfrak{m} \mathcal{O}_{Z}$.

The structure morphism $f: Y \rightarrow X$ factors as a sequence of projective morphisms

$$
Y \stackrel{g}{\rightarrow} Z \stackrel{h}{\rightarrow} X
$$

where $Y$ is the blow up the ideal sheaf $\mathfrak{m} \mathcal{O}_{Z}$. Define line bundles on $Y$ by $\mathcal{L}=g^{*} \mathcal{O}_{Z}(1)$ and $\mathcal{M}=\mathfrak{m} \mathcal{O}_{Y}$. Then $\mathcal{O}_{Y}(1) \cong \mathcal{M} \otimes \mathcal{L}$.

We have $\mathcal{O}_{Z}(1)\left|V_{j}=\bar{x}_{j} \mathcal{O}_{V_{j}}, \mathcal{L}\right| U_{i, j}=\bar{x}_{j} \mathcal{O}_{U_{i, j}}$ and $\mathcal{M} \mid U_{i, j}=u_{i} \mathcal{O}_{U_{i, j}}$.

We give three consequences (Proposition 2.1, Proposition 2.2 and Corollary 2.3) of Serre's fundamental theorem for projective morphisms which will be useful.

Proposition 2.1. $\bigoplus_{k \geq 0} H^{i}\left(Y, \mathcal{L}^{k}\right)$ are finitely generated $R[E]$-modules for all $i \in \mathbb{N}$.

Proof. Let $\widetilde{E^{k}}$ be the sheafication of $E^{k}$ on $X$. From the natural surjections for $k \geq 0$ of $\mathcal{O}_{Z}$-modules $g^{*}\left(\widetilde{E^{k}}\right) \rightarrow \mathcal{O}_{Z}(k)$, we obtain surjections $f^{*}\left(\widetilde{E^{k}}\right) \rightarrow \mathcal{L}^{k}$ of $\mathcal{O}_{Y}$-modules, and a surjection $f^{*}\left(\bigoplus_{k \geq 0} \widetilde{E^{k}}\right) \rightarrow \bigoplus_{k \geq 0} \mathcal{L}^{k}$. Hence $\bigoplus_{k \geq 0} \mathcal{L}^{k}$ is a finitely generated $f^{*}\left(\bigoplus_{k \geq 0} \widetilde{E^{k}}\right)$-module. By Theorem III.2.4.1 [8], $R^{i} f_{*}\left(\bigoplus_{k \geq 0} \mathcal{L}^{k}\right)$ is a finitely generated $\bigoplus_{k>0} \widetilde{E_{k}}$-module for $i \in \mathbb{N}$. Taking global sections on the affine $X$, we obtain the conclusions of the proposition.

Proposition 2.2. Suppose that $A$ is a Noetherian ring, and $B=\bigoplus_{k>0} B_{k}$ is a finitely generated graded A-algebra, which is generated by $B_{1}$ as an A-algebra. Let $C=\operatorname{Spec}(A)$ and $D=\operatorname{Proj}(B)$. Let $\alpha: D \rightarrow C$ be the structure morphism. Then there exists a positive integer $\bar{k}$ such that $B_{k}=\Gamma\left(D, \mathcal{O}_{D}(k)\right)$ for $k \geq \bar{k}$.

Proof. The ring $\bigoplus_{k \geq 0} \Gamma\left(D, \mathcal{O}_{D}(k)\right)$ is a finitely generated graded $B$-module by Theorem III.2.4.1 [8]. Hence $\left(\bigoplus_{k \geq 0} \Gamma\left(D, \mathcal{O}_{D}(k)\right)\right) / B$ is a finitely generated graded $B$ module. Since every element of this module is $B_{+}=\bigoplus_{k>0} B_{k}$ torsion, we have that $B_{k} / E_{k}=0$ for $k \gg 0$.

Taking the maximum over the $\bar{k}$ obtained from the above proposition applied to a finite affine cover of $W$, we obtain the following generalization of Proposition 2.2.

Corollary 2.3. Suppose that $W$ is a Noetherian scheme and $\mathcal{B}=\bigoplus_{k>0} \mathcal{B}_{k}$ is a finitely generated graded $\mathcal{O}_{W}$-algebra, which is locally generated by $\mathcal{B}_{1}$ as a $\mathcal{O}_{W}$ algebra. Let $W^{\prime}=\operatorname{Proj}(\mathcal{B})$ and let $\alpha: W^{\prime} \rightarrow W$ be the structure morphism. Then there exists a positive integer $\bar{k}$ such that $\mathcal{B}_{k}=\alpha_{*} \mathcal{O}_{W^{\prime}}(k)$ for $k \geq \bar{k}$, 


\section{Asymptotic Growth}

Proposition 3.1. Let $(R, \mathfrak{m})$ be a local domain of depth $\geq 2$. Let d be the dimension of $R$. Suppose that $E$ is a rank e R-submodule of a finitely generated free $R$-module $F$. Let notation be as above. Then there exist positive integers $k_{0}, k_{1}$ and $\tau$ such that

1) for $k \geq k_{0}, n \in \mathbb{Z}$ and $\mathfrak{p} \in X \backslash\{\mathfrak{m}\}$,

$$
\Gamma\left(Y, \mathcal{M}^{n} \otimes \mathcal{L}^{k}\right)_{\mathfrak{p}}=\left(E^{k}\right)_{\mathfrak{p}}
$$

2) For $k \geq k_{1}$,

$$
E^{k}:_{F^{k}} \mathfrak{m}^{\infty}=\Gamma\left(Y, \mathcal{M}^{-k \tau} \otimes \mathcal{L}^{k}\right)
$$

Proof. We first establish 1). $U_{i}=\operatorname{Spec}\left(R_{u_{i}}\right)$ for $1 \leq i \leq s$ is an affine cover of $X \backslash\{\mathfrak{m}\}$. $g \mid f^{-1}\left(U_{i}\right)$ is an isomorphism; in fact

$$
f^{-1}\left(U_{i}\right)=\operatorname{Proj}\left(R[\mathfrak{m} E]_{u_{i}}\right)=\operatorname{Proj}\left(R[E]_{u_{i}}\right)=h^{-1}\left(U_{i}\right) .
$$

By Proposition 2.2, there exist positive integers $a_{i}$ such that

$$
\Gamma\left(f^{-1}\left(U_{i}\right), \mathcal{M}^{-n} \otimes \mathcal{L}^{k}\right)=\Gamma\left(h^{-1}\left(U_{i}\right), \mathcal{O}_{Z}(k)\right)=\left(E^{k}\right)_{u_{i}}
$$

for $k \geq a_{i}$. Let $k_{0}=\max \left\{a_{1}, \ldots, a_{s}\right\}$. Then for $\mathfrak{p} \in U_{i}$

$$
\Gamma\left(Y, \mathcal{M}^{-n} \otimes \mathcal{L}^{k}\right)_{\mathfrak{p}}=\Gamma\left(f^{-1}\left(U_{i}\right), \mathcal{M}^{n} \otimes \mathcal{L}^{k}\right)_{\mathfrak{p}}=\left(E^{k}\right)_{\mathfrak{p}}
$$

for $k \geq k_{0}$, establishing 1 ).

We now establish 2). Suppose that $n \geq 0$, and $k \geq 0$. Suppose that $\sigma \in E^{k}: F^{k} \mathfrak{m}^{n}$. Let $i, j$ be such that $1 \leq i \leq s$ and $1 \leq j \leq t . \sigma \mathfrak{m}^{n} \subset E^{k}$ implies $u_{i}^{n} \sigma \in E^{k}$ which implies there is an expansion

$$
u_{i}^{n} \sigma=\sum_{n_{1}+\cdots+n_{t}=k} r_{n_{1}, \ldots, n_{t}} \bar{x}_{1}^{n_{1}} \cdots \bar{x}_{t}^{n_{t}}
$$

with $r_{n_{1}, \ldots, n_{t}} \in R$. Thus

$$
u_{i}^{n} \sigma=\bar{x}_{j}^{k}\left(\sum_{n_{1}+\cdots+n_{t}=k} r_{n_{1}, \ldots, n_{t}}\left(\frac{\bar{x}_{1}}{\bar{x}_{j}}\right)^{n_{1}} \cdots\left(\frac{\bar{x}_{t}}{\bar{x}_{j}}\right)^{n_{t}}\right),
$$

so that $\sigma \in u_{i}^{-n} \bar{x}_{j}^{k} R_{i, j}$. Thus

$$
\sigma \in \cap_{i, j} u_{i}^{-n} \bar{x}_{j}^{k} R_{i, j}=\Gamma\left(Y, \mathcal{M}^{-n} \otimes \mathcal{L}^{k}\right) .
$$

We have established that for $k \geq 0$ and $n \geq 0$,

$$
E^{k}:_{F^{k}} \mathfrak{m}^{n} \subset \Gamma\left(Y, \mathcal{M}^{-n} \otimes \mathcal{L}^{k}\right) .
$$

Recall that $S$ is a polynomial ring $S=R\left[y_{1}, \ldots, y_{\gamma}\right]$ over $R$, where $\gamma$ is the rank of $F$. Let $W=\operatorname{Proj}(S)$, with natural morphism $\alpha: W \rightarrow X$. Let $\mathcal{I}$ be the sheafication of the graded ideal $E S$ on $W$. We have expansions

$$
\bar{x}_{i}=\sum_{l=1}^{\gamma} f_{i l} y_{l}
$$

with $f_{i l} \in R$.

The inclusion $R[E] \subset S$ induces a rational map from $W$ to $Z$. 
Let $\beta: W^{\prime} \rightarrow W$ be the blow up of the ideal sheaf $\mathcal{I}$. Let $\mathcal{N}=\mathcal{I O}_{W^{\prime}}$ be the induced line bundle. $W^{\prime}$ has an affine cover $A_{i, j}=\operatorname{Spec}\left(T_{i j}\right)$ for $1 \leq i \leq t$ and $1 \leq j \leq \gamma$ with

$$
T_{i j}=R\left[\frac{y_{1}}{y_{j}}, \ldots, \frac{y_{\gamma}}{y_{j}}\right]\left[\frac{\bar{x}_{1}}{\bar{x}_{i}}, \ldots, \frac{\bar{x}_{t}}{\bar{x}_{i}}\right] .
$$

From the inclusions

$$
R_{i}=R\left[\frac{\bar{x}_{1}}{\bar{x}_{i}}, \ldots, \frac{\bar{x}_{t}}{\bar{x}_{i}}\right] \subset T_{i j}
$$

we have induced morphisms $A_{i, j} \rightarrow V_{i}=\operatorname{Spec}\left(R_{i}\right)$ which patch to give a morphism $\varphi: W^{\prime} \rightarrow Z$ which is a resolution of indeterminacy of the rational map from $W$ to $Z$.

We calculate for all $i, j$,

$$
\varphi^{*}\left(\mathcal{O}_{Z}(1)\right)\left|A_{i, j}=\bar{x}_{i} \mathcal{O}_{A_{i j}}=y_{j}\left(\sum_{l} f_{i, l} \frac{y_{l}}{y_{j}}\right) \mathcal{O}_{A_{i j}}=\left(\beta^{*} \mathcal{O}_{W}(1)\right) \mathcal{I}\right| A_{i, j},
$$

to see that

$$
\left(\beta^{*} \mathcal{O}_{W}(1)\right) \otimes \mathcal{N} \cong \varphi^{*} \mathcal{O}_{Z}(1) .
$$

By Corollary 2.3, there exists a positive integer $k_{1} \geq k_{0}$ such that $\beta_{*} \mathcal{N}^{k}=\mathcal{I}^{k}$ for $k \geq k_{1}$. From the natural inclusion $\mathcal{O}_{Z}(k) \subset \varphi_{*} \varphi^{*} \mathcal{O}_{Z}(k)$, we have by the projection formula that for $k \geq k_{1}$,

$$
\begin{aligned}
h_{*} \mathcal{O}_{Z}(k) & \subset h_{*} \varphi_{*}\left(\varphi^{*} \mathcal{O}_{Z}(k)\right)=\alpha_{*} \beta_{*}\left(\beta^{*} \mathcal{O}_{W}(k) \otimes \mathcal{N}^{k}\right) \\
= & \alpha_{*}\left[\mathcal{O}_{W}(k) \otimes \beta_{*} \mathcal{N}^{k}\right]=\alpha_{*}\left[\mathcal{O}_{W}(k) \otimes \mathcal{I}^{k}\right] \\
& \subset \alpha_{*} \mathcal{O}_{W}(k)=\widetilde{F^{k}}
\end{aligned}
$$

where $\widetilde{F^{k}}$ is the sheafication of the $R$-module $F$ on $X$. Now we have

$$
\begin{aligned}
\Gamma\left(Y, \mathcal{M}^{-n} \otimes \mathcal{L}^{k}\right) & =\Gamma\left(X, f_{*}\left(\mathcal{M}^{-n} \otimes \mathcal{L}^{k}\right)\right) \\
& \subset \Gamma\left(X \backslash\{\mathfrak{m}\}, f_{*}\left(\mathcal{M}^{-n} \otimes \mathcal{L}^{k}\right)\right)=\Gamma\left(X \backslash\{\mathfrak{m}\}, h_{*} \mathcal{O}_{Z}(k)\right) \\
& \subset \Gamma\left(X \backslash\{\mathfrak{m}\}, \widetilde{F^{k}}\right)=F^{k}
\end{aligned}
$$

since $R$, and hence the free $R$-module $F^{k}$, have depth $\geq 2$.

From (11), we deduce that for $k, n \geq 0$,

$$
\left((E S)^{k}: S \mathfrak{m}^{n} S\right) \cap F^{k}=E^{k}:_{F^{k}} \mathfrak{m}^{n} .
$$

By 1.5 [11] or Theorem 1.3 [16], there exists a positive integer $\tau$ such that

$$
(E S)^{k}:_{S} \mathfrak{m}^{k \tau} S=(E S)^{k}:_{S}(\mathfrak{m} S)^{\infty}
$$

for all $k \geq 0$. Thus from (15) we have that

$$
E^{k}:_{F^{k}} \mathfrak{m}^{k \tau}=E^{k}:_{F^{k}} \mathfrak{m}^{\infty}
$$

for $k \geq 0$. From (16), (12) and (14), we have inclusions

$$
E^{k}:_{F^{k}} \mathfrak{m}^{\infty} \subset \Gamma\left(Y, \mathcal{M}^{-k \tau} \otimes \mathcal{L}^{k}\right) \subset F^{k}
$$

for $k \geq k_{1}$. The conclusions of 2) of the proposition now follow from 1) of the proposition since $E^{k}:_{F^{k}} \mathfrak{m}^{\infty}$ is the largest $R$-submodule $N$ of $F^{k}$ which has the property that $N_{\mathfrak{p}}=\left(E^{k}\right)_{\mathfrak{p}}$ for $\mathfrak{p} \in X \backslash\{\mathfrak{m}\}$. 
Theorem 3.2. Suppose that $(R, \mathfrak{m})$ is a local domain of depth $\geq 2$ which is essentially of finite type over a field $K$ of characteristic zero. Let $d$ be the dimension of $R$. Suppose that $E$ is a rank e submodule of a finitely generated free $R$-module $F$. Then the limit

$$
\lim _{k \rightarrow \infty} \frac{\lambda\left(E^{k}:_{F^{k}} \mathfrak{m}^{\infty} / E^{k}\right)}{k^{d+e-1}} \in \mathbb{R}
$$

exists.

Proof. Let notation be as above.

First consider the short exact sequences

$$
0 \rightarrow \Gamma\left(Y, \mathcal{L}^{k}\right) / E^{k} \rightarrow E^{k}:_{F^{k}} \mathfrak{m}^{\infty} / E^{k} \rightarrow E^{k}:_{F^{k}} \mathfrak{m}^{\infty} / \Gamma\left(Y, \mathcal{L}^{k}\right) \rightarrow 0
$$

$\bigoplus_{k \geq 0} \Gamma\left(Y, \mathcal{L}^{k}\right)$ is a finitely generated $R[E]$-module by Lemma 2.1. By 1$)$ of Proposition 3.1 , the support of the $R$-module $\Gamma\left(Y, \mathcal{L}^{k}\right) / E^{k}$ is contained in $\{\mathfrak{m}\}$ for all $k$. Since $\left(\bigoplus_{k \geq 0} \Gamma\left(Y, \mathcal{L}^{k}\right)\right) / R[E]$ is a finitely generated $R[E]$-module, there is a positive integer $r$ such that $\mathfrak{m}^{r}\left(\Gamma\left(Y, \mathcal{L}^{k}\right) / E^{k}\right)=0$ for all $k$. Since $\operatorname{dim} R[E] / \mathfrak{m} R[E] \leq \operatorname{dim} R+$ rank $E-1=d+e-1$, and $R / \mathfrak{m}^{r}$ is an Artin local ring, we have that $\lambda\left(\Gamma\left(Y, \mathcal{L}^{k}\right) / E^{k}\right)$ is a polynomial of degree less than or equal to $d+e-2$ for $k \gg 0$ by the Hilbert-Serre theorem. Thus there exists a constant $\alpha$ such that $\lambda\left(\Gamma\left(Y, \mathcal{L}^{k}\right) / E^{k}\right) \leq \alpha k^{d+e-2}$ for all $k$. From (17), we are now reduced to showing that the limit

$$
\lim _{k \rightarrow \infty} \frac{\lambda\left(E^{k}:_{F^{k}} \mathfrak{m}^{\infty} / \Gamma\left(Y, \mathcal{L}^{k}\right)\right)}{k^{d+e-1}}
$$

exists, from which we will have

$$
\lim _{k \rightarrow \infty} \frac{\lambda\left(E^{k}:_{F^{k}} \mathfrak{m}^{\infty} / \Gamma\left(Y, \mathcal{L}^{k}\right)\right)}{k^{d+e-1}}=\lim _{k \rightarrow \infty} \frac{\lambda\left(E^{k}:_{F^{k}} \mathfrak{m}^{\infty} / E^{k}\right)}{k^{d+e-1}}
$$

Taking global sections of the short exact sequences

$$
0 \rightarrow \mathcal{L}^{k} \rightarrow \mathcal{M}^{-k \tau} \otimes \mathcal{L}^{k} \rightarrow \mathcal{M}^{-k \tau} \otimes \mathcal{L}^{k} \otimes\left(\mathcal{O}_{Y} / \mathfrak{m}^{k \tau} \mathcal{O}_{Y}\right) \rightarrow 0,
$$

we obtain by Proposition 3.1 left exact sequences

$$
0 \rightarrow E^{k}: F^{k} \mathfrak{m}^{\infty} / \Gamma\left(Y, \mathcal{L}^{k}\right) \rightarrow \Gamma\left(Y, \mathcal{M}^{-k \tau} \otimes \mathcal{L}^{k} \otimes\left(\mathcal{O}_{Y} / \mathfrak{m}^{k \tau} \mathcal{O}_{Y}\right)\right) \rightarrow H^{1}\left(Y, \mathcal{L}^{k}\right)
$$

for $k \geq k_{1}$.

Let $u_{1}, \ldots, u_{s}$ be generators of the ideal $\mathfrak{m}$, and set $U_{i}=\operatorname{Spec}\left(R_{u_{i}}\right)$, so that $\left\{U_{1}, \ldots, U_{s}\right\}$ is an affine cover of $X \backslash\{\mathfrak{m}\}$. Then $\mathcal{L} \mid f^{-1}\left(U_{i}\right)$ is ample, so there exist positive integers $b_{i}$ such that $R^{1} f_{*}\left(\mathcal{L}^{k}\right) \mid U_{i}=0$ for $k \geq b_{i}$. Let $k_{2}=\max \left\{b_{1}, \ldots, b_{s}\right\}$. We have that the support of $H^{1}\left(Y, \mathcal{L}^{k}\right)$ is contained in $\{\mathfrak{m}\}$ for $k \geq k_{2}$.

$\bigoplus_{k \geq 0} H^{1}\left(Y, \mathcal{L}^{k}\right)$ is a finitely generated $R[E]$-module by Lemma 2.1. Hence the submodule $M=\bigoplus_{k \geq k_{2}} H^{1}\left(Y, \mathcal{L}^{k}\right)$ is a finitely generated graded $R[E]$-module. We have that $\mathfrak{m}^{r} M=0$ for some positive integer $r$. Since

$$
\operatorname{dim} R[E] / \mathfrak{m} R[E] \leq \operatorname{dim} R+\operatorname{rank} E-1=d+e-1,
$$

and $R / \mathfrak{m}^{r}$ is an Artin local ring, we have that $\lambda\left(H^{1}\left(Y, \mathcal{L}^{k}\right)\right)$ is a polynomial of degree less than or equal to $d+e-2$ for $k \gg 0$ by the Hilbert-Serre theorem. Thus there exists a constant $c$ such that

$$
\lambda\left(H^{1}\left(Y, \mathcal{L}^{k}\right)\right) \leq c k^{d+e-2}
$$


for all $k \geq 0$. By consideration of (18) and (19), we are reduced to proving that the limit

$$
\lim _{k \rightarrow \infty} \frac{\lambda\left(H^{0}\left(Y, \mathcal{M}^{-k \tau} \otimes \mathcal{L}^{k} \otimes \mathcal{O}_{Y} / \mathfrak{m}^{k \tau} \mathcal{O}_{Y}\right)\right)}{k^{d+e-1}}
$$

exists.

If $R / \mathfrak{m}$ is algebraic over $K$, let $K^{\prime}=K$. If $R / \mathfrak{m}$ is transcendental over $K$, let $t_{1}, \ldots, t_{r}$ be a lift of a transcendence basis of $R / \mathfrak{m}$ over $K$ to $R$. The rational function field $K\left(t_{1}, \ldots, t_{r}\right)$ is contained in $R$. Let $K^{\prime}=K\left(t_{1}, \ldots, t_{r}\right)$. We have that $R / \mathfrak{m}$ is finite algebraic over $K^{\prime}$.

There exists an affine $K^{\prime}$-variety $X^{\prime}=\operatorname{Spec}(A)$ such that $R$ is the local ring of a closed point $\alpha$ of $X^{\prime}$, and $E$ extends to a submodule $E^{\prime}$ of $A^{\gamma}$, where $\gamma$ is the rank of the free $R$-module $F$. We then have an inclusion of graded $A$-algebras $A\left[E^{\prime}\right] \subset \operatorname{Sym}\left(A^{\gamma}\right)$ which extends $R[E]$. Identify $\mathfrak{m}$ with its extension to a maximal ideal of $A$. The structure morphism $Y^{\prime}=\operatorname{Proj}\left(A\left[\mathfrak{m} E^{\prime}\right]\right) \rightarrow X^{\prime}$ is projective and its localization at $\mathfrak{m}$ is $f: Y \rightarrow X$. Let $\bar{X}$ be a projective closure of $X^{\prime}$ and let $\tilde{Y}$ be a projective closure of $Y^{\prime} . X^{\prime}$ is an open subset of $\bar{X}$ and $Y^{\prime}$ is an open subset of $\tilde{Y}$. Let $\bar{Y} \rightarrow \tilde{Y}$ be the blow up of an ideal sheaf which gives a resolution of indeterminancy of the rational map from $\tilde{Y}$ to $\bar{X}$. We may assume that the morphism $\bar{Y} \rightarrow \tilde{Y}$ is an isomorphism over the locus where the rational map is a morphism, and thus an isomorphism over the subset $Y^{\prime}$ of $\tilde{Y}$. Let $\bar{f}: \bar{Y} \rightarrow \bar{X}$ be the resulting morphism. We now establish that $\bar{f}^{-1}\left(X^{\prime}\right)=Y^{\prime}$. Suppose that $p \in X^{\prime}$ and $q \in \bar{f}^{-1}(p)$. Let $V$ be a valuation ring of the function field $L$ of $\bar{Y}$ (which is also the function field of $Y^{\prime}$ ) which dominates the local ring $\mathcal{O}_{\bar{Y}, q}$. By assumption, $V$ dominates the local ring $\mathcal{O}_{X^{\prime}, p} . V$ dominates the local ring of a point on $Y^{\prime}$, by the valuative criterion for properness (Theorem II.4.7 [9]) applied to the proper morphism $Y^{\prime} \rightarrow X^{\prime}$. Since $V$ dominates the local ring of a unique point on $\bar{Y}$, we have that $q \in Y^{\prime}$.

After possibly replacing $\bar{Y}$ with the blow up of an ideal sheaf on $\bar{Y}$ whose support is disjoint from $Y^{\prime}$, we may assume that $\mathcal{L}$ extends to a line bundle on $\bar{Y}$ which we will also denote by $\mathcal{L}$. We will identify $\mathfrak{m}$ with its extension to the ideal sheaf of the point $\alpha$ on $\bar{X}$, and identify $\mathcal{M}$ with its extension $\mathfrak{m} \mathcal{O}_{\bar{Y}}$ to a line bundle on $\bar{Y}$. Let $\mathcal{A}$ be an ample divisor on $\bar{X}$. Then there exists $l>0$ such that $\mathcal{C}=\bar{f}^{*}\left(\mathcal{A}^{l}\right) \otimes \mathcal{L}$ is generated by global sections and is big.

Set $\mathcal{B}=\mathcal{C} \otimes \mathcal{M}^{-\tau}$. Tensor the short exact sequences

$$
0 \rightarrow \mathcal{M}^{k \tau} \rightarrow \mathcal{O}_{\bar{Y}} \rightarrow \mathcal{O}_{\bar{Y}} / \mathfrak{m}^{k \tau} \mathcal{O}_{\bar{Y}} \cong \mathcal{O}_{Y} / \mathfrak{m}^{k \tau} \mathcal{O}_{Y} \rightarrow 0
$$

with $\mathcal{B}^{k}$ to obtain the short exact sequences

$$
0 \rightarrow \mathcal{C}^{k} \rightarrow \mathcal{B}^{k} \rightarrow \mathcal{M}^{-k \tau} \otimes \mathcal{L}^{k} \otimes \mathcal{O}_{Y} / \mathfrak{m}^{k \tau} \mathcal{O}_{Y} \rightarrow 0
$$

for $k \geq 0$. Taking global sections, we have exact sequences

$$
0 \rightarrow H^{0}\left(\bar{Y}, \mathcal{C}^{k}\right) \rightarrow H^{0}\left(\bar{Y}, \mathcal{B}^{k}\right) \rightarrow H^{0}\left(Y, \mathcal{M}^{-k \tau} \otimes \mathcal{L}^{k} \otimes \mathcal{O}_{Y} / \mathfrak{m}^{k \tau} \mathcal{O}_{Y}\right) \rightarrow H^{1}\left(\bar{Y}, \mathcal{C}^{k}\right) .
$$

For a coherent sheaf $\mathcal{F}$ on $\bar{Y}$, let

$$
h^{i}(\bar{Y}, \mathcal{F})=\operatorname{dim}_{K^{\prime}} H^{i}(\bar{Y}, \mathcal{F}) .
$$


Since $\mathcal{C}$ is semiample (generated by global sections and big) and $\bar{Y}$ has dimension $d+e-1$, we have that

$$
\lim _{k \rightarrow \infty} \frac{h^{1}\left(\bar{Y}, \mathcal{C}^{k}\right)}{k^{d+e-1}}=0
$$

This follows for instance from [5]. Since $\bigoplus_{k>0} H^{0}\left(\bar{Y}, \mathcal{C}^{k}\right)$ is a finitely generated $K^{\prime}$ algebra of dimension $d+e$, as $\mathcal{C}$ is generated by global sections and is big (or by the Riemann Roch theorem and the vanishing theorem of [5]) we have that the limit

$$
\lim _{k \rightarrow \infty} \frac{h^{0}\left(\bar{Y}, \mathcal{C}^{k}\right)}{k^{d+e-1}} \in \mathbb{Q}
$$

exists. Since $\mathcal{B}$ is big, by the corollary to [6] given in Example 11.4.7 [14] or [3], we have that the limit

$$
\lim _{k \rightarrow \infty} \frac{h^{0}\left(\bar{Y}, \mathcal{B}^{k}\right)}{k^{d+e-1}} \in \mathbb{R}
$$

exists. From the sequence (21), we see that

$$
\lim _{k \rightarrow \infty} \frac{h^{0}\left(Y, \mathcal{M}^{-k \tau} \otimes \mathcal{L}^{k} \otimes \mathcal{O}_{Y} / \mathfrak{m}^{k \tau} \mathcal{O}_{Y}\right)}{k^{d+e-1}} \in \mathbb{R}
$$

exists. The conclusions of the theorem now follow from (20) and the formula

$$
\begin{aligned}
& h^{0}\left(Y, \mathcal{M}^{-k \tau} \otimes \mathcal{L}^{k} \otimes \mathcal{O}_{Y} / \mathfrak{m}^{k \tau} \mathcal{O}_{Y}\right)=\operatorname{dim}_{K^{\prime}} H^{0}\left(Y, \mathcal{M}^{-k \tau} \otimes \mathcal{L}^{k} \otimes \mathcal{O}_{Y} / \mathfrak{m}^{k \tau} \mathcal{O}_{Y}\right) \\
& \quad=\left[R / \mathfrak{m}: K^{\prime}\right] \lambda\left(H^{0}\left(Y, \mathcal{M}^{-k \tau} \otimes \mathcal{L}^{k} \otimes \mathcal{O}_{Y} / \mathfrak{m}^{k \tau} \mathcal{O}_{Y}\right)\right)
\end{aligned}
$$

Remark 3.3. The conclusions of Theorem 3.2 are also true if $K$ is a perfect field of positive characteristic and $R / \mathfrak{m}$ is algebraic over $K$. In this case we have that $K^{\prime}=K$ in the proof of Theorem 3.2. Let $\bar{K}$ be an algebraic closure of $K$. Since $K$ is perfect, $\bar{Y} \times_{K} \bar{K}$ is reduced, and to compute the limit, we reduce to computing the sections of the pullback of $\mathcal{B}^{k}$ on the disjoint union of the irreducible (integral) components of $\bar{Y} \times_{K} \bar{K}$. Fujita's approximation theorem is valid on varieties over an algebraically closed field of positive characteristic, as was shown by Takagi [17], from which the existence of the limit now follows.

Remark 3.4. Theorem 3.2 is proven for graded ideals in [3]. An example where the limit is an irrational number is given in [3]. The theorem is proven with the additional assumptions that $R$ is regular, $E=I$ is an ideal in $F=R$, and the singular locus of $\operatorname{Spec}(R / I)$ is $\mathfrak{m}$ in [4]. Kleiman [13] has proven Theorem 3.2 in the case that $E$ is a direct summand of $F$ locally at every nonmaximal prime of $R$.

Corollary 3.5. Suppose that $(R, \mathfrak{m})$ is a local domain of depth $\geq 2$ which is essentially of finite type over a field $K$ of characteristic zero. Let $d$ be the dimension of $R$. Suppose that $E$ is a rank e submodule of a finitely generated free $R$-module $F$. Then the limit

$$
\lim _{k \rightarrow \infty} \frac{(d+e-1) !}{k^{d+e-1}} \lambda\left(H_{\mathfrak{m}}^{0}\left(F^{k} / E^{k}\right)\right) \in \mathbb{R}
$$

exists. Thus the epsilon multiplicity $\varepsilon(E)$ of the module E, defined in [19] as a limsup, actually exists as a limit.

The example of [3] shows that $\varepsilon(E)$ may be an irrational number. 
Proof. The corollary is immediate from Theorem 3.2 and (9).

Remark 3.6. The conclusions of Corollary 3.5 are valid if $K$ is a perfect field of positive characteristic and $R / \mathfrak{m}$ is algebraic over $K$, by Remark 3.3.

\section{Extension to domains of dimension $\geq 2$.}

In this section, we prove extensions of Theorem 1.1 and Corollary 1.2 to domains of dimension $\geq 2$. Let notation be as in Section 2 .

Suppose that $R$ is a domain of dimension $d \geq 2$ with a dualizing module. By the Theorem of Finiteness, Theorem VIII.2.1 (and footnote) [7],

$$
\bar{R}=\Gamma\left(X \backslash\{\mathfrak{m}\}, \mathcal{O}_{X}\right)=\cap_{\mathfrak{p} \in X \backslash\{\mathfrak{m}\}} R_{\mathfrak{p}}
$$

is a finitely generated $R$-module, which lies between $R$ and its quotient field. Since $\bar{R} / R$ is $\mathfrak{m}$-torsion,

$$
\lambda_{R}(\bar{R} / R)<\infty .
$$

Let $\mathfrak{m}_{1}, \ldots, \mathfrak{m}_{\alpha}$ be the maximal ideals of $\bar{R}$ which lie over $\mathfrak{m}$. By our construction,

$$
0=H_{\mathfrak{m}}^{1}(\bar{R})=H_{\mathfrak{m} \bar{R}}^{1}(\bar{R})=\bigoplus_{i=1}^{\alpha} H_{\mathfrak{m}_{i} \bar{R}}^{1}(\bar{R})
$$

SO

$$
H_{\mathfrak{m}_{i} \bar{R}_{\mathfrak{m}_{i}}}^{1}\left(\bar{R}_{\mathfrak{m}_{i}}\right)=H_{\mathfrak{m}_{i} \bar{R}}^{1}(\bar{R}) \otimes_{\bar{R}} \bar{R}_{\mathfrak{m}_{i}}=0
$$

for $1 \leq i \leq \alpha$, and thus $\operatorname{depth}\left(\bar{R}_{\mathfrak{m}_{i}}\right) \geq 2$ for $1 \leq i \leq \alpha$.

Let $\bar{F}=F \otimes_{R} \bar{R}$ and $\bar{R}[\bar{F}]=\bigoplus_{k \geq 0} \bar{F}^{k}$, so that $\bar{F}^{k} \cong F^{k} \otimes_{R} \bar{R}$ for all $k$. Let $\bar{E}=\bar{R} E$ be the $\bar{R}$-submodule of $\bar{F}$ generated by $E$. Let $\bar{R}[\bar{E}]=\bigoplus_{k \geq 0} \bar{E}^{k}$ be the $\bar{R}$-subalgebra of $\bar{R}[\bar{F}]$ generated by $\bar{E}$.

Let $u_{1}, \ldots, u_{s}$ be generators of the ideal $\mathfrak{m}$. For $k \in \mathbb{N}$, let $\widetilde{E^{k}}$ be the sheafification of $E^{k}$ on $X=\operatorname{Spec}(R)$.

There are identities

$$
H^{0}\left(X \backslash\{\mathfrak{m}\}, \widetilde{E^{k}}\right)=\cap_{i=1}^{s}\left(E^{k}\right)_{u_{i}}=E^{k}: \bar{F}^{k} \mathfrak{m}^{\infty} .
$$

From the exact sequence of cohomology groups

$$
0 \rightarrow H_{\mathfrak{m}}^{0}\left(E^{k}\right) \rightarrow E^{k} \rightarrow H_{\mathfrak{m}}^{0}\left(X \backslash\{\mathfrak{m}\}, \widetilde{E^{k}}\right) \rightarrow H_{\mathfrak{m}}^{1}\left(E^{k}\right) \rightarrow 0,
$$

we deduce that we have isomorphisms of $R$-modules

$$
H_{\mathfrak{m}}^{1}\left(E^{k}\right) \cong E^{k}: \bar{F}^{k} \mathfrak{m}^{\infty} / E^{k}
$$

for $k \geq 0$. The same calculation for $F^{k}$ shows that

$$
H_{\mathfrak{m}}^{1}\left(F^{k}\right) \cong F^{k}: \bar{F}^{k} \mathfrak{m}^{\infty} / F^{k} .
$$

From the left exact local cohomology sequence

$$
0 \rightarrow H_{\mathfrak{m}}^{0}\left(F^{k} / E^{k}\right) \rightarrow H_{\mathfrak{m}}^{1}\left(E^{k}\right) \rightarrow H_{\mathfrak{m}}^{1}\left(F^{k}\right),
$$

we have that

$$
\left.H_{\mathfrak{m}}^{0}\left(F^{k} / E^{k}\right) \cong\left(E^{k}: \bar{F}^{k} \mathfrak{m}^{\infty}\right) \cap F^{k}\right) / E^{k}=E^{k}:_{F^{k}} \mathfrak{m}^{\infty} / E^{k} .
$$


Theorem 4.1. Suppose that $(R, \mathfrak{m})$ is a local domain of dimension $d \geq 2$ which is essentially of finite type over a field $K$ of characteristic zero (or over a perfect field $K$ such that $R / \mathfrak{m}$ is algebraic over $K$ ). Suppose that $E$ is a rank e submodule of a finitely generated free $R$-module $F$. Then the limit

$$
\lim _{k \rightarrow \infty} \frac{\lambda\left(E^{k}: \bar{F}^{k} \mathfrak{m}^{\infty} / E^{k}\right)}{k^{d+e-1}} \in \mathbb{R}
$$

exists.

Proof. Since $\bar{E}^{k}: \bar{F}^{k} \mathfrak{m}^{\infty} / \bar{E}^{k}$ are finitely generated $\mathfrak{m} \bar{R}$-torsion $\bar{R}$-modules, we have that

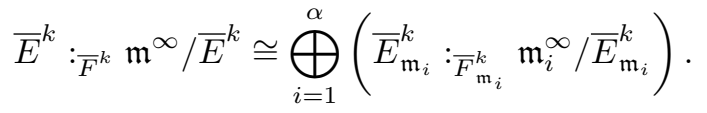

By Theorem 1.1, we have that

$$
\lim _{k \rightarrow \infty} \frac{\lambda_{\bar{R}_{\mathfrak{m}_{i}}}\left(\bar{E}_{\mathfrak{m}_{i}}^{k}: \bar{F}_{\mathfrak{m}_{i}}^{k} \mathfrak{m}_{i}^{\infty} / \bar{E}_{\mathfrak{m}_{i}}^{k}\right)}{k^{d+e-1}}
$$

exists for $1 \leq i \leq \alpha$. Since for any $\bar{R}_{\mathfrak{m}_{i}}$ module $M$ we have that

$$
\lambda_{R}(M)=\left[\bar{R} / \mathfrak{m}_{i}: R / \mathfrak{m}\right] \lambda_{\bar{R}_{\mathfrak{m}_{i}}}(M),
$$

we conclude that

$$
\lim _{k \rightarrow \infty} \frac{\lambda_{R}\left(\bar{E}^{k}: \bar{F}^{k} \mathfrak{m}^{\infty} / \bar{E}^{k}\right)}{k^{d+e-1}}
$$

exists. We have

$$
\bar{E}^{k}: \bar{F}^{k} \mathfrak{m}^{\infty}=\cap_{i=1}^{s}\left(\bar{E}^{k}\right)_{u_{i}}=\cap_{i=1}^{s}\left(E^{k}\right)_{u_{i}}=E^{k}: \bar{F}^{k} \mathfrak{m}^{\infty} .
$$

Consider the short exact sequences

$$
0 \rightarrow \bar{E}^{k} / E^{k} \rightarrow E^{k}: \bar{F}^{k} \mathfrak{m}^{\infty} / E^{k} \rightarrow \bar{E}^{k}: \bar{F}^{k} \mathfrak{m}^{\infty} / \bar{E}^{k} \rightarrow 0 .
$$

Now $\bar{R}[\bar{E}] / R[E]$ is a finitely generated $R[E]$-module, and the support of the $R$-module $\bar{E}^{k} / E^{k}$ is contained in $\{\mathfrak{m}\}$ for all $k$, so there exists a positive integer $r$ such that $\mathfrak{m}^{r}$ annihilates $\bar{R}[\bar{E}] / R[E]$. Thus (as in the argument following equation (17) in the proof of Theorem 3.2), we have that there exists a constant $\beta$ such that

$$
\lambda_{R}\left(\bar{E}^{k} / E^{k}\right) \leq \beta k^{d+e-2}
$$

for all $k$. The conclusions of the proposition now follow from (29), (31) and (30).

I thank Craig Huneke, Bernd Ulrich and Javid Validashti for pointing out the following consequence of Theorem 4.1.

Corollary 4.2. Suppose that $(R, \mathfrak{m})$ is a local domain of dimension $d \geq 2$ which is essentially of finite type over a field $K$ of characteristic zero (or over a perfect field $K$ such that $R / \mathfrak{m}$ is algebraic over $K$ ). Suppose that $E$ is a rank e submodule of a 
finitely generated free $R$-module $F$. Suppose that $\gamma=\operatorname{rank}(F)<d+e$. Then the limits

$$
\lim _{k \rightarrow \infty} \frac{\lambda\left(E^{k}:_{F^{k}} \mathfrak{m}^{\infty} / E^{k}\right)}{k^{d+e-1}} \in \mathbb{R}
$$

and

$$
\lim _{k \rightarrow \infty} \frac{(d+e-1) !}{k^{d+e-1}} \lambda\left(H_{\mathfrak{m}}^{0}\left(F^{k} / E^{k}\right)\right) \in \mathbb{R}
$$

exist. In particular, the epsilon multiplicity $\varepsilon(E)$ of $E$ exists as a limit.

Proof. We will establish that the limit (32) exists. We have exact sequences

$$
0 \rightarrow E^{k}: F^{k} \mathfrak{m}^{\infty} / E^{k} \rightarrow E^{k}: \bar{F}^{k} \mathfrak{m}^{\infty} / E^{k} \rightarrow E^{k}: \bar{F}^{k} \mathfrak{m}^{\infty} / E^{k}:_{F^{k}} \mathfrak{m}^{\infty} \rightarrow 0
$$

and inclusions

for $k \geq 0$.

We have

$$
F^{k}: \bar{F}^{k} \mathfrak{m}^{\infty} / F^{k}=\bar{F}^{k} / F^{k} \cong(\bar{R} / R)^{\left(\begin{array}{c}
k+\gamma-1 \\
\gamma-1
\end{array}\right)} .
$$

Since $\gamma=\operatorname{rank}(F)<d+e$, we have

$$
\lim _{k \rightarrow \infty} \frac{\lambda_{R}\left(E^{k}: \bar{F}^{k} \mathfrak{m}^{\infty} / E^{k}:_{F^{k}} \mathfrak{m}^{\infty}\right)}{k^{d+e-1}}=0 .
$$

The existence of the limit (32) now follows from (34) and Theorem 4.1. The existence of the limit (33) is immediate from (32) and (27).

\section{References}

[1] W. Bruns and J. Herzog, Cohen-Macaulay rings, Cambridge University Press, 1993.

[2] A. Crabbe, D. Katz, J. Striuli, E. Theodorescu, Hilbert Samuel polynomials of the contravariant extension functor, Nagoya Math. J. 198 (2010), 1-22.

[3] S.D. Cutkosky, H.T. Ha, H. Srinivasan and E. Theodorescu, Asymptotic behaviour of the length of local cohomology, Canad. J. Math. 57 (2005), 1178 - 1192.

[4] S.D. Cutkosky, J. Herzog and H. Srinivasan, Asymptotic growth of algebras associated to powers of ideals, Math. Proc. Camb. Philos. Soc. 148 (2010), $55-72$.

[5] T. Fujita, Semipositive line bundles, J. of the Faculty of Science, The University of Tokyo 30 (1983), 353 - 378.

[6] T. Fujita, Approximating Zariski decomposition of line bundles, Kodai Math. J. 17 (1994), 1 - 3.

[7] A. Grothendieck, Cohomologie locale des faisceaux coherents et theoremes de Lefschetz locaux et globaux, North-Holland, Amsterdam (1968).

[8] A. Grothendieck and J. Dieudonné, Étude cohomologique des faisceaus cohérents, Publ. Math. IHES 11 (1961).

[9] R. Hartshorne, Algebraic Geometry, Springer Verlag, 1977.

[10] J. Herzog, T. Puthenpurakal, J. Verma, Hilbert polynomials and powers of ideals, Math. Proc. Cambridge Math. Soc. 145 (2008), 623 - 642.

[11] D. Katz and S. McAdam, Two asymptotic functions, Comm. in Alg. 17 (1989) 1069 1091.

[12] D. Katz and E. Theodorescu, Hilbert polynomials for the extension function, J. Algebra 319 (2008), $2319-2336$.

[13] S. Kleiman, The $\varepsilon$-multiplicity as a limit, communication to the author. 
[14] R. Lazarsfeld, Positivity in algebraic geometry, Springer Verlag, Berlin, 2004.

[15] R. Lazarsfeld and M. Mustata, Convex bodies associated to linear series, Ann. Sci. Ec. Norm. Super. 42 (2009), 783 - 835.

[16] I. Swanson, Powers of ideals: primary decomposition, Artin-Rees lemma and regularity, Math. Annalen 307 (1997), 299 - 313.

[17] S. Takagi, Fujita's approximation theorem in positive characteristics, J. Math. Kyoto Univ, 47 (2007), 179 - 202

[18] E. Theodorescu, Derived functors and Hilbert polynomials, Math. Proc. Cambridge Philos. Soc. 132 (2002), $75-88$.

[19] B. Ulrich and J. Validashti, Numerical criteria for integral dependence, to appear in Math. Proc. Camb. Phil. Soc.

Department of Mathematics, University of Missouri, Columbia, MO 65211, USA

E-mail address: cutkoskys@missouri.edu 